\title{
Algunas reflexiones sobre el concepto de desastre natural
}

Some reflections on the concept of natural disaster

Algumas reflexões sobre o conceito de desastre natural

Quelques réflexions sur la notion de catastrophe naturelle

\section{Fernando Antonio Ignacio González}

\section{(2) OpenEdition}

Journals

\section{Edición electrónica}

URL: https://journals.openedition.org/espacoeconomia/21037

DOI: 10.4000/espacoeconomia.21037

ISSN: 2317-7837

\section{Editor}

Núcleo de Pesquisa Espaço \& Economia

\section{Referencia electrónica}

Fernando Antonio Ignacio González, «Algunas reflexiones sobre el concepto de desastre natural», Espaço e Economia [Online], 22 | 2021, posto online no dia 09 janeiro 2022, consultado o 11 agosto 2022. URL: http://journals.openedition.org/espacoeconomia/21037 ; DOI: https://doi.org/10.4000/ espacoeconomia.21037

Este documento fue generado automáticamente el 11 agosto 2022.

\section{cc) (1) (ㅇ)}

Creative Commons - Atribuição-NãoComercial-Compartilhalgual 4.0 Internacional - CC BY-NC-SA 4.0 https://creativecommons.org/licenses/by-nc-sa/4.0/ 


\title{
Algunas reflexiones sobre el concepto de desastre natural
}

\author{
Some reflections on the concept of natural disaster \\ Algumas reflexões sobre o conceito de desastre natural \\ Quelques réflexions sur la notion de catastrophe naturelle
}

Fernando Antonio Ignacio González

\section{Introducción}

1 Los desastres naturales constituyen una seria amenaza a nivel global. En las últimas dos décadas (1998-2018) han ocasionado más de 1.2 millones de muertes y más de 3.3 billones de dólares en pérdidas directas (Munich Re, 2020). Además, la ocurrencia de desastres se asocia a un menor crecimiento económico, un reducido logro educativo y un empeoramiento en la salud de las personas afectadas (Bustelo et al., 2012; Lazzaroni y van Bergeijk, 2013; Klomp y Valckx, 2014; Karim y Noy, 2016; Dias de Oliveira, 2018; Hyland y Russ, 2019; González et al., 2020; 2021). A futuro, se espera un incremento en la frecuencia y severidad en la ocurrencia de desastres naturales a consecuencia del cambio climático (Intergovernmental Panel on Climate Change [IPCC], 2018).

2 La ocurrencia de desastres naturales no es homogénea entre países. Algunas localizaciones presentan un mayor nivel de riesgo ambiental (ejemplo: zonas costeras o de elevada actividad sísmica). La distribución de sus impactos tampoco es homogénea. Los países en desarrollo, y en general las personas pobres, tienden a resultar más afectados ante la ocurrencia de desastres (Klomp y Valckx, 2014; Hallegatte et al., 2020). Esto es esperable al contemplar la existencia de una infraestructura precaria especialmente en términos de vivienda-, falta de acceso a servicios básicos $\mathrm{y}$, en general, una menor cantidad de recursos para hacer frente y reponerse al desastre. Lo anterior indica la posible existencia de un círculo vicioso entre desastres naturales y pobreza: las personas pobres tienden a localizarse en espacios de mayor riesgo ambiental dado que frecuentemente son más accesibles en términos económicos; y a su 
vez, la ocurrencia de desastres agrava la situación de pobreza al reducir los recursos de los hogares y su capacidad para generarlos (Hallegatte et al., 2020).

La evidencia empírica confirma lo anterior. A pesar que el número de desastres es similar en países desarrollados y en desarrollo, la mayor parte de las muertes ocurre en países del segundo grupo (Hu et al., 2018; Centre for Research on the Epidemiology of Disasters [CRED], 2015). De todos los desastres naturales que tuvieron lugar, en 2015, el $44 \%$ ocurrió en países de ingresos bajos, a la vez que el $68 \%$ de los fallecimientos por desastres pertenecen a estos países (CRED, 2015). Esto es, cada desastre causa, en promedio, un $50 \%$ más de muertes en países de ingresos bajos que en aquellos de ingresos altos.

En años recientes se ha observado un creciente interés en investigadores de diferentes disciplinas por el estudio de los desastres naturales y sus efectos sobre las comunidades afectadas. Esto parece ser consecuencia de la ocurrencia de desastres naturales de amplia magnitud -como el terremoto de Sumatra-Andamán (2004) y el huracán Katrina (2005)- (Okuyama, 2007). En forma más reciente, otros desastres naturales también han ganado amplio interés entre investigadores; esto incluye -pero no se limita a- los terremotos de Haití (enero de 2010) y Chile (febrero de 2010) y el terremoto y tsunami de Japón (marzo de 2011). El gráfico 1 refleja la evolución anual de la cantidad de investigaciones publicadas en relación a este tópico.

Gráfico 1: Documentos publicados, por año, en la temática de desastres (1998-2019)

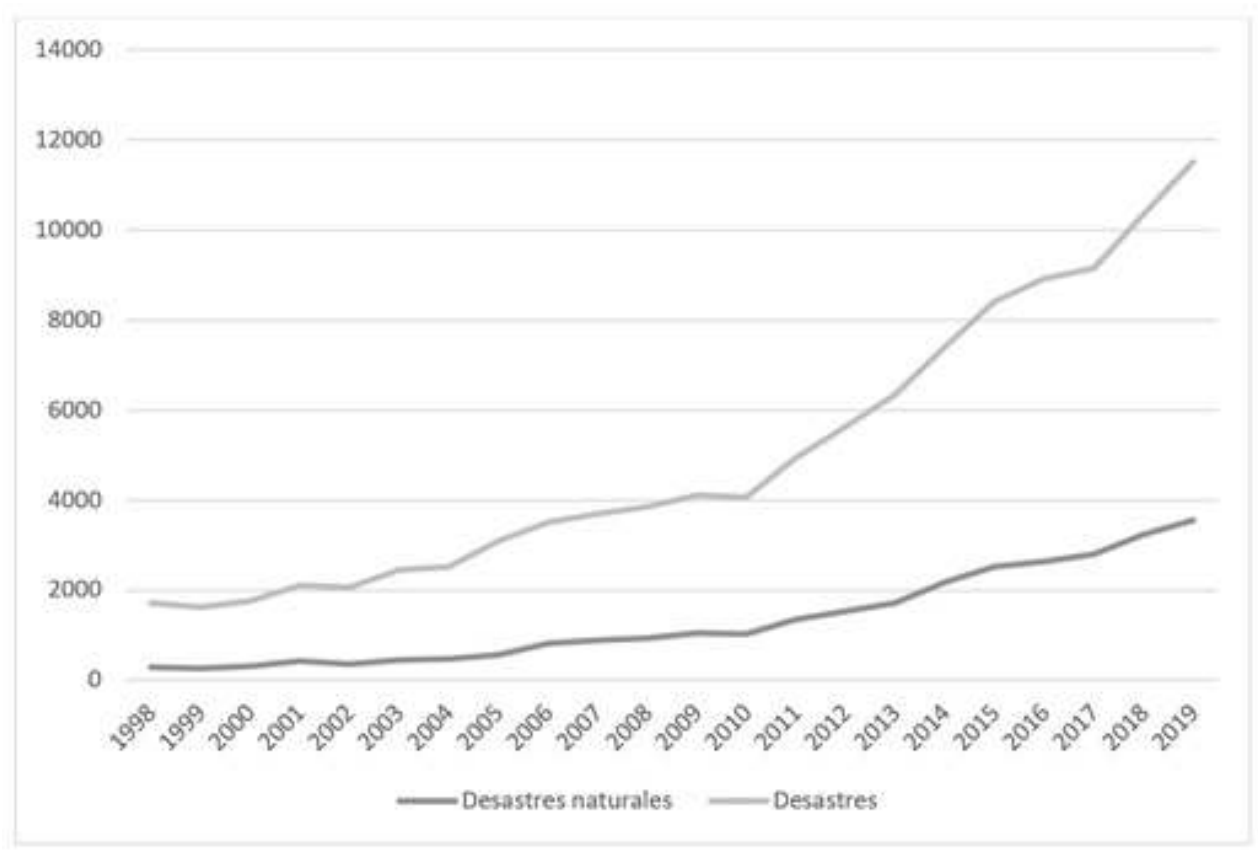

Fuente: elaboración propia en base a Science Direct. Nota: la búsqueda se realizó considerando, individualmente, los descriptores "disasters" y "natural disasters".

5 A pesar de su relevancia, dado sus potenciales efectos socio-económicos, la literatura de desastres no ha logrado alcanzar un consenso en términos de qué se considera desastre natural y qué no (Ratti, 2017). Su definición varía entre disciplinas y en el tiempo (Perry, 2018). Al respecto, se ha señalado que los desastres pueden ser más fácilmente reconocidos que definidos (Britton, 1986). Lo anterior resulta especialmente relevante 
considerando que se requiere una adecuada identificación de los desastres ocurridos y los riesgos asociados a los efectos de la implementación de políticas públicas de preparación, mitigación y reparación frente a desastres naturales. Además, una definición de desastre es fundamental para poder identificar sus causas, elementos y consecuencias.

Una definición de desastre que ha obtenido amplia difusión en la literatura es aquella propuesta por la Oficina de Naciones Unidas para la Reducción del Riesgo de Desastres (UNISDR por sus siglas en inglés). Este organismo sostiene que un desastre es "una seria interrupción en el funcionamiento de una comunidad o sociedad debido a eventos amenazantes interactuando con condiciones de exposición, vulnerabilidad y capacidad, y que generan una o más de los siguientes: pérdidas e impactos humanos, materiales, económicos y ambientales" (UNISDR, 2018). Típicamente las definiciones de desastre han hecho uso de la noción de estrés colectivo (Barton 1970; Haas y Drabek, 1973) según la cual las demandas al sistema superan su capacidad para dar respuesta sin alterar su estructura. También es posible identificar, a priori, múltiples elementos constitutivos del desastre, los cuales serán examinados a lo largo de este trabajo: amenaza (evento natural subyacente), exposición, vulnerabilidad y capacidad. La literatura reciente ha señalado que los desastres naturales se relacionan más estrechamente con aspectos políticos, sociales y económicos que con la amenaza natural. Así, la vulnerabilidad es el resultado de la desigualdad en el acceso a los recursos, discriminación y falta de poder político (Bolin y Stanford, 1998). Al respecto, Perry (2018) señala la existencia de tres etapas en la conceptualización de desastres: en la primera, denominada enfoque clásico, se concibe al desastre como fuera del control o influencia humana. La segunda etapa, enfoque de desastre-amenaza, enfatiza las interacciones entre el evento natural subyacente y la comunidad afectada; mientras que la tercera etapa, desastre como fenómeno social, se ha volcado ampliamente en el análisis de los determinantes socioeconómicos -en lugar de físicos o naturales- de la ocurrencia y consecuencias de los desastres.

7 En este contexto, en este trabajo se avanza con una discusión conceptual acerca de qué se entiende por desastre natural, vulnerabilidad y riesgo a desastres. En particular, se re-examina la evolución de la literatura de desastres y en como ésta fue alterando el foco del análisis desde el evento natural subyacente (amenaza natural) hacia las condiciones socio-económicas pre-existentes en la comunidad afectada (vulnerabilidad). Los resultados sugieren que la noción de desastre natural es una construcción social y continúa siendo materia de debate cuál es la mejor terminología a utilizar: mientras que es frecuente la denominación de desastre natural, esta expresión es cuestionada dado que parece indicar -erróneamente- que el desastre es exclusiva responsabilidad de la naturaleza y la conducta humana no posee influencia.

Excede al objetivo de este trabajo generar un inventario exhaustivo de definiciones de desastre natural. En su lugar, en este trabajo se busca capturar las principales definiciones generadas en este tópico. Siguiendo a Perry (2018), las definiciones aquí incluidas son aquellas ideadas por científicos sociales con propósitos teóricos. Los antecedentes incluidos surgen de la búsqueda por palabras claves en un reconocido motor de búsqueda ("natural disasters" y "conceptualization", en ScienceDirect). Además, fueron relevados los sitios web de instituciones con amplia experiencia en el estudio de desastres (CEPAL, CRED, CEPREDENAC, FEMA y Banco Mundial). Luego de la lectura de título y resumen, fueron incluidos aquellos trabajos que avanzan con 
propuestas teóricas sobre el concepto de desastre. Este trabajo añade valor a la literatura de desastres de dos formas. Primero, sintetiza la evidencia referida a la evolución temporal del concepto de desastre natural y sus elementos constitutivos. Segundo, sintetiza la literatura referida a riesgo de desastres.

\section{Desastres naturales, vulnerabilidad y riesgo}

9 Frecuentemente, diferentes acepciones son empleadas para referirse a una situación de desastre (catástrofe, cataclismo, emergencia, calamidad, etc.) a la vez que existen múltiples definiciones (Ratti, 2017). Resulta esencial, entonces, una clara delimitación de qué es, y no es, un desastre natural. Una definición ampliamente difundida es la propuesta por la Oficina de Naciones Unidas para la Reducción del Riesgo de Desastres (UNISDR por sus siglas en inglés) donde se sostiene que un desastre es "una seria interrupción en el funcionamiento de una comunidad o sociedad debido a eventos amenazantes interactuando con condiciones de exposición, vulnerabilidad y capacidad, y que generan una o más de los siguientes: pérdidas e impactos humanos, materiales, económicos y ambientales" (UNISDR, 2018). Esta definición se sustenta en la existencia de un evento amenazante que desencadena el desastre. Este evento recibe el nombre de amenaza.

Según la amenaza sea consecuencia directa del accionar humano o de la naturaleza el desastre resultante puede ser clasificado como tecnológico/antropogénico o natural, respectivamente (Below et al., 2009). En el primer grupo se encuentran los accidentes industriales o de transporte. Los desastres naturales, por su parte, se clasifican en seis grupos: geofísicos, meteorológicos, hidrológicos, climatológicos, biológicos y extraterrestres. Esta distinción entre desastres naturales y tecnológicos se emplea a los fines de clarificar qué tipos de desastres se consideran en cada caso según el proceso inmediato (amenaza) que los desencadena. De ningún modo pretende desconocer la potencial influencia del accionar humano sobre el ambiente y en cómo esto puede afectar la frecuencia e intensidad de los desastres. El uso de la expresión desastre natural se halla ampliamente extendida entre la literatura de desastres, aunque esto puede resultar problemático. Chmutina y von Meding (2019) argumentan que resulta preferible el uso de la expresión desastre (en lugar de desastre natural) y una clara identificación de los tipos de amenaza que se pretenden analizar. De esta forma, se evitaría la concepción -errónea- de que los desastres resultantes de amenazas naturales son exclusiva responsabilidad de la naturaleza y no del accionar humano.

11 Desde una perspectiva socio-económica la sola existencia de una amenaza natural (evento geofísico, meteorológico, hidrológico, climatológico, biológico o extra-terrestre subyacente) no configura la ocurrencia de un desastre natural. Esta amenaza natural, únicamente, se transforma en un desastre natural desde el momento en que genera alguna consecuencia social, económica o política negativa sobre la comunidad afectada y que supone una regresión o retraso en su desarrollo (Herzer et al., 2004). Esto es, debe existir una condición de exposición a la amenaza por parte de algún elemento del subsistema socio-económico. En otras palabras, la existencia de una amenaza natural es una condición necesaria pero no suficiente para la ocurrencia de un desastre natural. Así, una tormenta eléctrica que tenga lugar en una zona desértica sin generar daños en personas ni activos no es considerada un desastre natural. 

implica una interacción entre la amenaza y condiciones de vulnerabilidad. Esto indica que la presencia de una misma amenaza puede generar consecuencias muy disímiles dependiendo de las condiciones de vulnerabilidad o de la capacidad de hacer frente y reponerse -luego del desastre- de las personas o activos expuestos. Así, un evento (ej. terremoto) con las mismas características (alcance, duración, intensidad) puede dar lugar a un mayor impacto negativo en una zona cuyas viviendas o edificios no sean aptos para este tipo de eventos -en relación a la misma situación en una zona cuya infraestructura se encuentra mejor preparada-. El concepto de vulnerabilidad en el contexto de desastres naturales ha sido ampliamente discutido por la literatura.

tablas 1 y 2, a continuación, resumen diferentes definiciones de desastre y vulnerabilidad, respectivamente. De la Tabla 1 se observa que las definiciones de lo que constituye un desastre han tendido a considerar dos elementos centrales: existencia de una amenaza (evento potencialmente peligroso) y la efectivización de las consecuencias negativas derivadas de este evento. Llamativamente, algunas de estas definiciones resaltan el carácter repentino del desastre (Barton, 1969; Barkun, 1974) lo cual, en principio excluiría a eventos naturales de evolución lenta (ej. las sequías).

Desde la propuesta de Britton (1986) es que el concepto de vulnerabilidad se menciona explícitamente en la definición de desastre (Villalibre Calderón, 2013). Aquí se reconoce que la efectivización de las consecuencias negativas derivadas de la amenaza no resulta independiente del entorno socio-económico en el cual tiene lugar. En otras palabras, es necesario que la comunidad afectada presente algún grado de vulnerabilidad ante la ocurrencia de una amenaza dado que, de lo contrario, no se produciría ninguna consecuencia negativa y el evento aislado no calificaría como desastre. Lo anterior implica aceptar que son las condiciones de desarrollo de una comunidad las que determinan la interrupción de sus funciones o su nivel de destrucción y que, por ende, el concepto de desastre es una categoría social (Herzer et al., 2004). Esta consideración integral, natural y socio-económica, no implica abandonar el estudio del ámbito físiconatural de la amenaza subyacente sino adoptar una perspectiva más amplia que incluya el examen de los mecanismos sociales que crean riesgos desiguales para personas de una comunidad (Bolin y Stanford, 1998).

Tabla 1: Definiciones de desastre en publicaciones académicas

\begin{tabular}{|l|l|}
\hline Autor & Definición \\
\hline Fritz (1961) & $\begin{array}{l}\text { Evento, concentrado en tiempo y espacio, en el cual una sociedad o una subdivisión } \\
\text { de ésta enfrenta un severo peligro e incurre en pérdidas de tal magnitud -en } \\
\text { personas o pertenencias físicas- que el sistema social es interrumpido y se evita el } \\
\text { cumplimiento de algunas o todas de las funciones esenciales de la sociedad }\end{array}$ \\
\hline Barton (1969) & $\begin{array}{l}\text { Cambios repentinos y violentos en el ambiente físico que amenazan la vida y la } \\
\text { propiedad }\end{array}$ \\
\hline Barkun (1974) & $\begin{array}{l}\text { Interrupción severa, repentina e inesperada de los acuerdos estructurales } \\
\text { normales dentro de un sistema o subsistema social, resultante de una fuerza sobre } \\
\text { la cual el sistema no posee un control firme }\end{array}$ \\
\hline
\end{tabular}




\begin{tabular}{|c|c|}
\hline $\begin{array}{l}\text { Quarantelli } \\
(1985)\end{array}$ & $\begin{array}{l}\text { Ocasión de crisis de consenso en la que las demandas del sistema humano exceden } \\
\text { la capacidad de respuesta del sistema }\end{array}$ \\
\hline Britton (1986) & $\begin{array}{l}\text { Ruptura generalizada, temporaria o no, en los procesos sociales establecidos, } \\
\text { rutinas e interacciones que conlleva a cambio sociales colectivos y que interactúa } \\
\text { con condiciones de vulnerabilidad }\end{array}$ \\
\hline $\begin{array}{l}\text { Romero } \quad y \\
\text { Maskrey } \\
(1993)\end{array}$ & $\begin{array}{l}\text { Correlación entre fenómenos naturales peligrosos y determinadas condiciones } \\
\text { socioeconómicas y físicas vulnerables }\end{array}$ \\
\hline $\begin{array}{l}\text { Wilches- } \\
\text { Chaux (1993) }\end{array}$ & $\begin{array}{l}\text { Pérdidas materiales y humanas derivadas de la Inhabilidad de un sistema para } \\
\text { absorber mediante cambios grandes o pequeños en sus estructuras los cambios del } \\
\text { sistema superior }\end{array}$ \\
\hline CRID (2001) & $\begin{array}{l}\text { Seria interrupción en el funcionamiento de una sociedad causando vastas pérdidas } \\
\text { a nivel humano, material o ambiental, suficientes para que la sociedad afectada no } \\
\text { pueda salir adelante por sus propios medios }\end{array}$ \\
\hline CEPAL (2003) & $\begin{array}{l}\text { Suceso violento, inesperado o de ejecución lenta, que puede causar pérdidas de } \\
\text { vidas humanas, aflicción en la sociedad -o en una parte de ella-, un desajuste } \\
\text { temporal en los sistemas de vida prevalecientes, así como daños materiales y } \\
\text { dificultados en el funcionamiento de la sociedad y la economía }\end{array}$ \\
\hline ADPC (2004) & $\begin{array}{l}\text { Evento amenazante que causa una cantidad inaceptablemente alta de muertes o de } \\
\text { daño a las propiedad. }\end{array}$ \\
\hline $\begin{array}{l}\text { CEPREDENAC } \\
(2007)\end{array}$ & $\begin{array}{l}\text { Situación, contexto o proceso social que se desencadena como resultado de la } \\
\text { manifestación de un fenómeno peligroso de origen natural, socio-natural o } \\
\text { antropogénico que, al encontrar condiciones propicias de vulnerabilidad en una } \\
\text { población y en su estructura productiva e infraestructura, causa alteraciones } \\
\text { intensas, graves y extendidas en las condiciones normales de funcionamiento del } \\
\text { país, región, zona, o comunidad afectada, las cuales no pueden ser enfrentadas o } \\
\text { resueltas de manera autónoma utilizando los recursos disponibles }\end{array}$ \\
\hline CEPAL (2014) & $\begin{array}{l}\text { Consecuencia de fenómenos naturales desencadenantes de procesos que provocan } \\
\text { daños físicos y pérdidas de vidas humanas y de capital, al tiempo que alteran la } \\
\text { vida de comunidades y personas, y la actividad económica de los territorios } \\
\text { afectados }\end{array}$ \\
\hline $\begin{array}{l}\text { Kumar et al. } \\
(2014)\end{array}$ & $\begin{array}{l}\text { Evento adverso causado por un proceso natural de la Tierra y cuyas consecuencias } \\
\text { incluyen pérdidas humanas, de cultivos y de animales }\end{array}$ \\
\hline CRED (2020) & $\begin{array}{l}\text { Situación o evento que sobrepasa la capacidad local y requiere un pedido de ayuda } \\
\text { nacional o internacional. Evento repentino e imprevisto que causa gran daño, } \\
\text { destrucción y sufrimiento humano }\end{array}$ \\
\hline FEMA (2020) & $\begin{array}{l}\text { Ocurrencia de una catástrofe natural que resulta en severos daños a la propiedad, } \\
\text { mortalidad o múltiples heridas. Un gran desastre es aquel que excede la capacidad } \\
\text { de respuesta de la jurisdicción local y requiere ayuda estatal o federal }\end{array}$ \\
\hline
\end{tabular}



dos aspectos centrales: sensibilidad/susceptibilidad y capacidad de respuesta del sistema o comunidad afectados. La sensibilidad se refiere al grado en que la comunidad es modificada o afectada por la amenaza. La capacidad de respuesta es la habilidad para ajustarse o resistir la amenaza y recuperarse, moderando los daños potenciales (CEPAL, 2003). Además, se concibe que la vulnerabilidad no surge como consecuencia de la amenaza natural, sino que es una característica interna pre-existente.

Los conceptos de exposición y vulnerabilidad (incluyendo a la susceptibilidad y la capacidad) son nociones relacionadas, pero no idénticas. Así, ante una inundación los hogares pobres podrían verse más afectados dado que, frecuentemente, se localizan en zonas de mayor riesgo ambiental (ej: a orillas de cursos de agua) -es decir, tienen una mayor exposición-. Además, dado que sus viviendas tenderán a estar constituidas por materiales más precarios y con un menor acceso a servicios básicos tienen mayores chances de sufrir consecuencias negativas (mayor susceptibilidad). Finalmente, dado que los hogares pobres poseen una menor cantidad de recursos les resultará más difícil la reconstrucción de sus activos afectados (menor capacidad de respuesta).

Tabla 2: Definiciones de vulnerabilidad en publicaciones académicas

\begin{tabular}{|c|c|}
\hline Autor & Definición \\
\hline UNDRO (1979) & $\begin{array}{l}\text { Es una medida de la pérdida de un elemento o un conjunto de elementos en riesgo } \\
\text { resultante de la ocurrencia de un fenómeno natural de una cierta magnitud y } \\
\text { expresado en un escala de } 0 \text { (ningún daño) a } 1 \text { (pérdida total) }\end{array}$ \\
\hline Bogard (1989) & Inhabilidad de implementar medidas efectivas para asegurarse contra las pérdidas \\
\hline $\begin{array}{l}\text { Wilches- } \\
\text { Chaux (1993) }\end{array}$ & $\begin{array}{l}\text { Incapacidad de una comunidad para absorber, mediante el autoajuste, los efectos } \\
\text { de un determinado cambio en su medio ambiente }\end{array}$ \\
\hline $\begin{array}{l}\text { Bohle et al. } \\
(1994)\end{array}$ & $\begin{array}{l}\text { Medida agregada del bienestar humano que combina la exposición ambiental, } \\
\text { social, económica y política a un rango de perturbaciones potencialmente dañinas }\end{array}$ \\
\hline Campos (1998) & $\begin{array}{l}\text { Configuración total de condiciones objetivas y subjetivas de existencia, } \\
\text { históricamente determinadas y protagonizadas por sujetos colectivos concretos, } \\
\text { que originan o acentúan su predisposición ante ciertas amenazas y potencializan la } \\
\text { acción agresora de esta últimas }\end{array}$ \\
\hline $\begin{array}{l}\text { CEPAL y } \text { BID } \\
(2000)\end{array}$ & $\begin{array}{l}\text { Probabilidad de que una comunidad, expuesta a una amenaza natural, según el } \\
\text { grado de fragilidad de sus elementos, pueda sufrir daños materiales o humanos }\end{array}$ \\
\hline $\begin{array}{l}\text { Cardona } \\
(2001)\end{array}$ & $\begin{array}{l}\text { Predisposición o susceptibilidad física, económica, política o social que tiene una } \\
\text { comunidad de ser afectada o de sufrir daños en caso que un fenómeno } \\
\text { desestabilizador, natural o antrópico, se manifieste. }\end{array}$ \\
\hline Lavell (2001) & $\begin{array}{l}\text { Características diferenciadas de la sociedad, o sus subconjuntos, que le } \\
\text { predisponen a sufrir daños frente a un evento físico externo, y que dificultan su } \\
\text { posterior recuperación }\end{array}$ \\
\hline
\end{tabular}




\begin{tabular}{|l|l|}
\hline CEPAL (2003) & $\begin{array}{l}\text { Características y circunstancias de una comunidad o sistema que los vuelve } \\
\text { susceptibles a los efectos dañinos de una amenaza natural }\end{array}$ \\
\hline $\begin{array}{l}\text { Wisner et al. } \\
(2003)\end{array}$ & $\begin{array}{l}\text { Características de una persona o grupo y su situación que influencian su capacidad } \\
\text { de anticiparse, afrontar, resistir y recuperarse del impacto de una amenaza natural }\end{array}$ \\
\hline $\begin{array}{l}\text { CEPREDENAC } \\
(2007)\end{array}$ & $\begin{array}{l}\text { Factor de riesgo interno de un elemento o grupo de elementos expuestos a una } \\
\text { política o social que tiene una comunidad de ser afectada o de sufrir efectos } \\
\text { adversos en caso de que se manifieste un fenómeno peligroso de origen natural, } \\
\text { socio natural o antropogénico. Representa también las condiciones que } \\
\text { imposibilitan o dificultan la recuperación autónoma posterior. }\end{array}$ \\
\hline $\begin{array}{l}\text { Susman et al. } \\
(2020)\end{array}$ & $\begin{array}{l}\text { Grado en el cual las diferentes clases de una sociedad se hallan diferencialmente en } \\
\text { riesgo }\end{array}$ \\
\hline
\end{tabular}

Fuente: elaboración propia

21 La literatura también ha resaltado la estrecha relación entre la vulnerabilidad a desastres y la pobreza (CEPAL, 2014). En efecto, resulta intuitivo pensar que aquellos hogares localizados en zonas de alto riesgo ambiental, con viviendas precarias, falta de acceso a servicios básicos y a información relevante y con presencia de individuos con privaciones nutricionales tendrán mayores chances de sufrir daño y les resultará más dificultosa su recuperación -en relación a hogares que no experimenten estas privaciones-. La literatura ha indicado que las personas pobres experimentan un mayor estrés luego del desastre, tienen un menor capital social para hacer frente el shock y enfrentan mayores dificultades en conseguir una nueva vivienda (Bolin, 1986). En este sentido, la relación entre vulnerabilidad y pobreza es aún más estrecha si se concibe a la pobreza desde una perspectiva multidimensional.

La pobreza multidimensional constituye una concepción que se aparta de las típicas mediciones que consideran, exclusivamente, a la falta de ingresos para identificar una situación de pobreza (pobreza por ingresos o monetaria). En su lugar, una concepción multidimensional define la presencia de múltiples privaciones de distintas dimensiones del bienestar: calidad de la vivienda, educación, empleo, ingresos, protección social, servicios básicos, etc. Lo anterior implica que aquellos hogares o personas multidimensionalmente pobres son también más propensos a sufrir daños ante la ocurrencia de un evento natural extremo.

Por lo anterior, la existencia de una situación de desastre requiere la presencia de tres elementos: un evento natural potencialmente peligroso (amenaza), una comunidad -o parte de ella- en el marco de la cual tenga lugar este evento (exposición) y la susceptibilidad a sufrir daños derivados de este evento natural (vulnerabilidad). La conjunción de estos tres elementos (amenaza, exposición y vulnerabilidad) define lo que se denomina riesgo de desastre.

24 El riesgo de desastre es concebido como una medida probabilística de las potenciales pérdidas de vidas, heridos, o activos dañados o destruidos que podrían tener lugar en un sistema, comunidad o sociedad en un período específico de tiempo (UNISDR, 2018). La Tabla 3 resume algunas de las principales definiciones de riesgo de desastre provistas por la literatura académica. 
Tabla 3: Conceptualizaciones de riesgo vinculado a desastres naturales

\begin{tabular}{|c|c|c|}
\hline Autor & Concepto de riesgo & $\begin{array}{l}\text { Operacionalización } \\
{[\text { Riesgo }=f(\cdot)]}\end{array}$ \\
\hline $\begin{array}{l}\text { IUGS } \\
(1997)\end{array}$ & $\begin{array}{l}\text { Medida de la probabilidad y severidad de un efecto adverso } \\
\text { a la vida, salud, propiedad o el ambiente. }\end{array}$ & $\begin{array}{l}f(\text { Amenaza, } \quad \text { Pérdida } \\
\text { potencial })\end{array}$ \\
\hline $\begin{array}{l}\text { Hahn et al } \\
(2003)\end{array}$ & $\begin{array}{l}\text { Probabilidad de consecuencias dañinas o pérdidas } \\
\text { esperadas que resulta de las interacciones entre las } \\
\text { amenazas naturales y las condiciones de vulnerabilidad/ } \\
\text { capacidad }\end{array}$ & $\begin{array}{l}\text { f(Amenaza, Exposición, } \\
\text { Vulnerabilidad, } \\
{\text { Capacidad })^{a}}^{\text {a }}\end{array}$ \\
\hline $\begin{array}{l}\text { Wisner et } \\
\text { al (2003) }\end{array}$ & $\begin{array}{l}\text { Función compuesta de la amenaza natural y el número de } \\
\text { personas, caracterizadas por su distinto grado de } \\
\text { vulnerabilidad a la amenaza considerada, que ocupan el } \\
\text { espacio en el momento de la amenaza }\end{array}$ & $\begin{array}{l}f(\text { Amenaza, } \\
\text { Vulnerabilidad })\end{array}$ \\
\hline $\begin{array}{l}\text { ISDR } \\
(2004)\end{array}$ & $\begin{array}{l}\text { Probabilidad de consecuencias perjudiciales o pérdidas } \\
\text { esperadas resultado de interacciones entre amenazas } \\
\text { naturales y condiciones de vulnerabilidad }\end{array}$ & $\begin{array}{l}f(\text { Amenaza, } \\
\text { Vulnerabilidad, } \\
\text { Exposición) }\end{array}$ \\
\hline $\begin{array}{l}\text { Smith } \quad y \\
\text { Petley } \\
(2009)\end{array}$ & $\begin{array}{l}\text { Probabilidad de ocurrencia de una amenaza que ocasione } \\
\text { pérdidas }\end{array}$ & $f($ Probabilidad, Pérdida) \\
\hline $\begin{array}{l}\text { UNU-EHS } \\
(2011)\end{array}$ & $\begin{array}{l}\text { Interacción compleja entre amenaza natural y factores } \\
\text { políticos, sociales y ambientales }\end{array}$ & $\begin{array}{l}f(\text { Exposición, } \\
\text { Vulnerabilidad }^{\mathrm{b}}\end{array}$ \\
\hline $\begin{array}{l}\text { Gilles } \\
(2012)\end{array}$ & $\begin{array}{l}\text { Conjunción de un fenómeno natural -amenaza- y la } \\
\text { vulnerabilidad de las personas y propiedades expuestas }\end{array}$ & $\begin{array}{l}f(\text { Amenaza, } \\
\text { Vulnerabilidad) }\end{array}$ \\
\hline $\begin{array}{l}\text { ICSU } \\
(2015)\end{array}$ & $\begin{array}{l}\text { Probabilidad, en un período de tiempo específico, de } \\
\text { impactos adversos debido a eventos climáticos extremos } \\
\text { interactuando con condiciones sociales vulnerables }\end{array}$ & $\begin{array}{l}f(\text { Amenaza, } \\
\text { Vulnerabilidad, } \\
\text { Exposición) }\end{array}$ \\
\hline
\end{tabular}

Fuente: elaboración propia. ${ }^{a}$ Los componentes del riesgo se combinan en forma aditiva y Capacidad disminuye los niveles de riesgo. ${ }^{b}$ Vulnerabilidad incluye tres subcomponentes (susceptibilidad, capacidad de afrontamiento y capacidad de adaptación).

Se observa una multiplicidad de concepciones de riesgo de desastre (probabilidad, función compuesta, interacción compleja, conjunción). No obstante, en todos los casos, el análisis transcurre entre la esfera natural/ambiental (probabilidad de ocurrencia, intensidad o pérdidas esperadas por la amenaza) y la esfera socio-económica (vulnerabilidad, exposición y/o capacidad de afrontamiento de las personas o comunidades afectadas). Por ello, el nivel de riesgo, para diferentes amenazas naturales, puede diferir de una localización a otra y entre personas de una misma comunidad. Además, frecuentemente se considera al riesgo de desastre como un resultado multiplicativo -por oposición a aditivo- de sus componentes (amenaza, exposición y vulnerabilidad). Lo anterior indica que la ausencia de alguno de estos 
elementos conlleva a una situación de riesgo 0 . Esto es, si no tiene lugar alguna amenaza natural, o no existen elementos de una comunidad expuestos, o no se presenta un estado de vulnerabilidad, el riesgo de desastre es nulo.

Debe ser remarcado que, en años recientes, un creciente consenso se ha desarrollado en relación a la consideración simultánea de múltiples amenazas naturales en la evaluación del riesgo de desastre (UNISDR, 2005; Kappes, 2011; UNU-EHS, 2011; Liu, 2015), dando lugar al análisis del riesgo multi-amenaza. En particular, el análisis del riesgo multi-amenaza -entendido como el tratamiento conjunto de todas las amenazas relevantes en un contexto específico (Kappes, 2011)- impone complejidades metodológicas adicionales, considerando que se debe armonizar bajo una misma métrica una multiplicidad de eventos (geológicos, meteorológicos, etc.) donde cada uno posee sus propios factores disparadores, áreas de incidencia y escalas de severidad (Liu, 2015). Ventajosamente, considerar el riesgo multi-amenaza permite un examen integral del fenómeno, además que posibilita identificar las posibles interacciones entre amenazas (ejemplo: una inundación generada a partir de una tormenta). Usualmente, se ha estudiado el efecto domino existente entre amenazas (Marzocchi et al., 2012).

\section{Conclusiones}

En este trabajo se ha analizado cuáles son los elementos que la literatura ha, frecuentemente, identificado como necesarios para la existencia de un desastre natural (amenaza natural, exposición y vulnerabilidad) y cómo la conjunción de estos elementos da lugar al denominado riesgo de desastre. En particular, se ha observado que la efectivización de daños o pérdidas -a partir de la interacción entre la amenaza natural y la comunidad expuesta en condiciones de vulnerabilidad- da lugar al desastre.

Lo anterior implica aceptar que un desastre natural es, en última instancia, una categoría social. En este sentido, el foco del análisis se ha movido desde el examen de la amenaza natural (evento geofísico, hidrológico, climatológico, meteorológico, biológico o extraterrestre subyacente) hacia el análisis de sus consecuencias socio-económicas. En particular, la existencia de condiciones de vulnerabilidad -y su interrelación con la presencia de múltiples privaciones simultáneas- ha ganado centralidad. Desde una perspectiva de política pública, esto sugiere un mayor interés por acciones de preparación y mitigación -en lugar de únicamente actividades de reparación ex-post.

31 En un contexto de cambio climático en donde se espera un incremento en la frecuencia y severidad en la ocurrencia de desastres naturales, alcanzar una adecuada definición e identificación de lo que constituye un desastre resulta esencial para optimizar los esfuerzos de preparación, mitigación y reparación. Al respecto, aun es materia de debate cuál es la mejor denominación (desastre, desastre natural, calamidad, catástrofe, amenaza natural, peligro natural). En cualquier caso, considerando los elementos constitutivos (amenaza, exposición, vulnerabilidad) -a futuro- los esfuerzos también deben estar dirigidos hacia alcanzar una más amplia y profunda medición de las consecuencias socio-económicas generadas por un desastre natural. Una precisa delimitación de lo que constituye un desastre en conjunto con robusta información acerca de su alcance y consecuencias permitirá una mejor comprensión de su impacto micro y macro económicos y la implementación de acciones de política pública efectivas. 


\section{BIBLIOGRAFÍA}

BARKUN, M. Disaster and the Millenium. New Haven: Yale University Press, 1974.

BARTON, A. Communities in disasters: a sociological analysis of collective stress situations. New York: Doubleday, 1969.

BARTON, A. Communities in disaster: A sociological analysis of collective stress situations. Garden City: Double-day, 1970

BELOW, R., WIRTZ, A., GUHA-SAPIR, D. Disaster Category Classification and peril Terminology for Operational Purposes. Universite Catholique de Louvain Doc. No 264, 2009. Disponible en: https://www.cred.be/node/564

BOGARD, W. Bringing social theory to hazards research: conditions and consequences of the mitigation of environmental hazards. Sociological Perspectives, 31(2), 147-168, 1989.

BOHLE, H., DOWNING, T., WATTS, M. Climate change and social vulnerability: towards a sociology and geography of food security. Global Environmental Change, 4(1), 37-48, 1994.

BOLIN, R. The 1986 Californian Floods. Research Report 2, Natural Hazards Research and Applications Information Center, 1986.

BOLIN, R., STANFORD, L. The Northridge Eartquake: vulnerability and disaster. London and New York: Routledge, 1998.

BRITTON, N. Developing and understanding of disasters. ANZJS, 22(2), 254-271, 1986.

BUSTELO, M., ARENDS-KUENNING, M., LUCHETTI, L. Persistent impact of natural disasters on child nutrition and schooling: evidence from the 1999 Colombian Earthquake. Working paper 6354, IZA, 2012.

CAMPOS, A. Educación y prevención de desastres. Working paper, Fondo de Naciones Unidas para la Infancia, Facultad Latinoamericana de Ciencias Sociales y Red de Estudios Sociales en Prevención de Desastres en América Latina, 1998.

CARDONA, O. La necesidad de repensar de manera holística los conceptos de vulnerabilidad y riesgo: una crítica y una revisión necesaria para la gestión. Trabajo presentado en la conferencia International Work-Conference on Vulnerability in Disaster Theory and Practice, junio de 2001Holanda.

CEPAL. Manual para la evaluación del impacto socioeconómico y ambiental de desastres, 2003. Reporte disponible en: https://repositorio.cepal.org/bitstream/handle/11362/2781/ S2003652_es.pdf

CEPAL. Manual para la evaluación de desastres, 2014. Reporte disponible en: https:// repositorio.cepal.org/handle/11362/35894

CEPAL y BID. Un tema del desarrollo: la reducción de la vulnerabilidad frente a los desastres. Working paper LC/MEX/L.428, Comisión Económica para América Latina y el Caribe y Banco Interamericano de Desarrollo, 2000.

CEPREDENAC. Glosario actualizado de términos en la perspectiva de la reducción de riesgo a desastres. Reporte del Centro de Coordinación para la Prevención de Desastres Naturales en América Central, 2007. Disponible en: https://www.cepredenac.org/documentos 
CHMUTINA, K., VON MEDING, J. A Dilemma of Language: "Natural Disasters" in Academic Literature. International Journal of Disaster Risk Reduction, 10, 283-292, 2019.

CRED. The human cost of natural disasters 2015. Report of Centre for Research on the Epidemiology of Disasters, 2015.

CRED. EM-DAT Glossary, 2020. Disponible en: https://www.emdat.be/Glossary\#letter_d

CRID. Vocabulario controlado sobre desastres. Reporte del Centro Regional de Información de Desastres, 2001. Disponible en: http://cidbimena.desastres.hn/docum/crid/ListAlfabdeTerm.pdf DIAS DE OLIVEIRA, L. Sustentabilidade em questão: Lições acerca do Rompimento da Barragem em Mariana (MG, 2015). Espaço e Economia, 13, 2018. doi.org/10.4000/espacoeconomia.4041.

FEMA. Glossary of terms. Federal Emergency Management Agency, 2020. Disponible en: https:// www.fema.gov/pdf/plan/glo.pdf

FRITZ, C. Disaster en Merton, R.; Nisbet, R. (eds). Contemporary Social Problems, New York: Brace and World, 1961.

GILLES, A. Natural hazard mapping across the world. A comparative study between a social approach and an economic approach to vulnerability. Cybergeo: European Journal of Geography, Environment, Nature, Landscape, documento 602, 2012.

GONZÁLEZ, F. Natural disasters and mortality: A probabilistic analysis for Argentina (1970-2015). Revista de Estudios Andaluces, 40, 78-95, 2020.

GONZÁLEZ, F., SANTOS, M., LONDON, S. Persistent effects of natural disasters on human development: quasi-experimental evidence for Argentina. Environment, Development and Sustainability, 2020. https://doi.org/10.1007/s10668-020-01064-7

GONZÁLEZ, F., SANTOS, M., LONDON, S. Multidimensional Poverty and Natural Disasters in Argentina (1970-2010). Journal of Human Development and Capabilities, in press, 2021.

GONZÁLEZ, F., DIP, J., LONDON, S. Long-lasting Effects of Pandemics: The Case of the 1918 Influenza Pandemic in Argentina. Spatial and Spatio-Temporal Epidemiology, in press, 2021.

HAAS, E., DRABEK, T. Complex organizations: A sociological perspective. New York: McMillan, 1973.

HAHN, H., VILLAGRÁN DE LEÓN, J., HIDAJAT, R. Indicators and other disaster risk management instruments for communities and local Governments. Banco Interamericano de Desarrollo y Agencia Alemana para la Cooperación Internacional, 2003.

HALLEGATTE, S., VOGT-SCHILB, A., ROZENBERG, J., BANGALORE, M., BEAUDET, C. From Poverty to Disaster and Back: A Review of the Literature. Economics of Disasters and Climate Change, 4 , 223-247, 2020.

HERZER, H., CAPUTO, M., CELIS, A. Gestión de desastre ENSO en América Latina: Propuesta de Consolidación de un Red Regional de Investigación Comparativa, Información y Capacitación desde una Perspectiva Social. Informe Final Argentina, CENTRO estudios sociales y ambientales, 2004. Disponible en: https://cambio-global.org/enso/informes/anho4/Argentina/index.html HU, P., ZHANG, Q., SHI, P., CHEN, B., FANG, J. Flood-induced mortality across the globe: Spatiotemporal pattern and influencing factors. Science of the Total Environment, 643, 171-182, 2018.

HYLAND, M., RUSS, J. Water as destiny-The long-term impacts of drought in sub-Saharan Africa. World Development, 115, 30-45, 2019. 
ICSU. Disasters risk research and assessment to promote risk reduction and management. Reporte del International Council for Science e International Social Science Council, 2015. Disponible en: http://www.iugg.org/policy/Report_RiskReduction_WCDRR_2015.pdf

IPCC. Impacts of $1.5^{\circ} \mathrm{C}$ Global Warming on Natural and Human Systems, en: Global Warming of $1.5^{\circ} \mathrm{C}$. An IPCC Special Report on the impacts of global warming of $1.5^{\circ} \mathrm{C}$ above pre-industrial levels and related global greenhouse gas emission pathways, in the context of strengthening the global response to the threat of climate change, sustainable development, and efforts to eradicate poverty, 2018. Disponible en: https://www.ipcc.ch/sr15/

ISDR. Living with risk: A global review of disaster reduction initiatives. Ginebra: ONU, 2004. IUGS. Quantitative risk assessment for slopes and landslides-the state of the art. En Cruden, D.; Fell, R. Landslide risk assessment. Rotterdam: Balkema, 1997.

KAPPES, M. Multi-Hazard Risk Analyses: Concept and its Implementation. Tesis doctoral, Universidad de Viena, 2011.

Karim, A., Noy, I. Poverty and natural disasters-A qualitative survey of the empirical literature. The Singapure Economic Review, 61(1), 2016. https://doi.org/10.1142/S0217590816400014

KlOMP, J., VALCKX, K. Natural disasters and economic growth: A meta-analysis. Global Environmental Change, 26, 183-195, 2014.

KUMAR, A., BRAHMANAND, P., NAYAK, A. Management of cyclone disaster in agriculture sector in coastal areas. Working paper 117, Directorate of Water Management, 2014.

LAVELL, A. Sobre la Gestión de riesgos: Apuntes hacia una definición. Working paper, Centro de Información sobre Desastres y Salud Biblioteca Médica Nacional, 2001.

LAZZARONI, S., VAN BERGEIJK, P. Natural disasters impact, factors of resilience and development: A meta-analysis of the macroeconomic literatura. Documento de trabajo 554, ISS, 2013.

LIU, B. Modelling multi-hazard risk assessment: A case study in the Yangtze River Delta, China. Tesis doctoral, School of Earth and Environment, University of Leeds, 2015.

MARZOCCHI, W., GARCIA-ARISTIZABAL, A., GASPARINI, P., MASTELLONE, M., DI RUOCCO, A. Basic principles of multi-risk assessment: a case study in Italy. Natural Hazards, 62(2), 551-573, 2012.

MUNICH RE. Natural catastrophe service, 2020. Disponible en: https:// natcatservice.munichre.com/overall/1 (Acceso en junio de 2020).

OKUYAMA, Y. Economic modeling for disaster impact analysis: Past, present, and future. Economic Systems Research, 19(2), 115-124, 2007.

PERRY, R. Defining disaster: an evolvind concept. En Rodriguez, H.; Donner, W.; Trainor, J. (Eds.). Handbook of Disaster Research, 2nd Edition, Switzerland: Springer International Publishing, 2018.

QUARANTELLI, E. What is a disaster? The need for clarification in definition and conceptualization in research. Working paper 177, University of Delaware, 1985.

RATTI, M. The Economics of Natural Disasters: an overview of the current research issues and methods. Working paper 3, CERE, 2017.

ROMERO, G., MASKREY, A. Como entender los desastres naturales en MASKREY, A. (ed). Los desastres no son naturales. Reporte de la Red de Estudios Sociales en Prevención de Desastres en América Latina (LA RED), 1993. 
SMITH, K., PETLEY, D. Environmental hazards: assessing risk and reducing disaster. Londes y Nueva York: Routledge, 2009.

SUSMAN, P., O'KEEFE, P., WISNER, B. Global disasters, a radical interpretation en Hewitt, K. (ed). Interpretations of calamity from the viewpoint of human ecology. Londres y Nueva York: Routledge, 2020.

UNDRO. Natural disasters and vulnerability analysis. Reporte de la Reunión del Grupo de Expertos, Office of the United Nations Disaster Relief Co-ordinator, 1979.

UNISDR. Terminology on disaster risk reduction, 2018. Disponible en: https://www.undrr.org/ terminology/disaster

UNU-EHS. World Risk Report 2011. Reporte de United Nations University e Institute for Environmental and Human Security, 2011. Disponible en: https://collections.unu.edu/eserv/ UNU:2046/WorldRiskReport-2011_online_EN.pdf

VILLLIBRE CALDERÓN, C. Concepto de urgencia, emergencia, catástrofe y desastre: revisión histórica y bibliográfica. Tesis de Magister en Análisis y Gestión de Emergencia y Desastre, Universidad de Oviedo, 2013.

WILCHES-CHAUX, G. La vulnerabilidad global en Maskrey, A. (ed). Los desastres no son naturales. Reporte de la Red de Estudios Sociales en Prevención de Desastres en América Latina (LA RED), 1993.

WISNER, B., BLAIKIE, P., CANNON, T., DAVIS, I. At risk: natural hazards, people's vulnerability and disasters, Parte I Segunda Edición. Londres y New York: Routledge, 2003.

\section{RESÚMENES}

El actual contexto de cambio climático indica que, a futuro, la frecuencia y severidad en la ocurrencia de desastres naturales aumentará, al igual que sus consecuencias socio-económicas. En este trabajo se busca re-examinar la literatura de desastres desde una perspectiva conceptual. Se discute qué se entiende por desastre natural, cuáles son sus elementos constitutivos y cómo evolucionó este concepto. Los hallazgos contribuyen a la literatura de desastres al sintetizar la evolución teórica de este tópico. Los resultados sugieren que, en los últimos 15 años, la temática de desastres naturales ha ganado un creciente interés entre investigadores a la par de la ocurrencia de desastres de amplia magnitud. A su vez, el concepto de desastre natural ha evolucionado desde una perspectiva más enfocada en el análisis del entorno físico-natural hacia una perspectiva socio-natural. Así, surge que el concepto de desastre natural es, en última instancia, una categoría social.

The current climate change context indicates that, in the future, the frequency and severity of natural disasters will increase, as will their socio-economic consequences. This work seeks to reexamine the disaster literature from a conceptual perspective. It is discussed what is understood by natural disaster, what are its constituent elements and how this concept evolved. The findings contribute to the disaster literature by synthesizing the theoretical evolution of this topic. The results suggest that, in the last 15 years, the subject of natural disasters has gained increasing interest among researchers along with the occurrence of large-scale disasters. In turn, the concept of natural disaster has evolved from a perspective more focused on the analysis of the physical-natural environment towards a socio-natural perspective. Thus, it emerges that the concept of natural disaster is, ultimately, a social category. 
O contexto atual das mudanças climáticas indica que, no futuro, a frequência e a gravidade dos desastres naturais aumentarão, assim como suas consequências socioeconômicas. Este artigo busca reexaminar a literatura sobre desastres de uma perspectiva conceitual. Discute-se o que se entende por desastre natural, quais são seus elementos constituintes e como esse conceito evoluiu. As descobertas contribuem para a literatura sobre desastres ao sintetizar a evolução teórica deste tópico. Os resultados sugerem que, nos últimos 15 anos, o tema desastres naturais vem ganhando cada vez mais interesse entre os pesquisadores junto com a ocorrência de desastres de grande porte. Por sua vez, o conceito de desastre natural evoluiu de uma perspectiva mais focada na análise do ambiente físico-natural para uma perspectiva sócio-natural. Assim, verifica-se que o conceito de desastre natural é, em última instância, uma categoria social.

Le contexte actuel du changement climatique indiqué qu'à l'avenir, la fréquence et la gravité des catastrophes naturelles augmenteront, tout comme leurs conséquences socio-économiques. Cet article cherche à réexaminer la littérature sur les catastrophes d'un point de vue conceptuel. On discute de ce que l'on entend par catastrophe naturelle, de ses éléments constitutifs et de l'évolution de ce concept. Les résultats contribuent à la littérature sur les catastrophes en synthétisant l'évolution théorique de ce sujet. Les résultats suggèrent qu'au cours des 15 dernières années, le sujet des catastrophes naturelles a suscité un intérêt croissant parmi les chercheurs, parallèlement à la survenue de catastrophes à grande échelle. À son tour, le concept de catastrophe naturelle a évolué d'une perspective davantage axée sur l'analyse de l'environnement physique-naturel vers une perspective socio-naturelle. Ainsi, il apparaît que le concept de catastrophe naturelle est, en définitive, une catégorie sociale.

\section{ÍNDICE}

Palavras-chave: desastres naturais, vulnerabilidade, risco

Mots-clés: catastrophes naturelles, vulnérabilité, risqué

Keywords: natural disasters, vulnerability, risk

Palabras claves: desastres naturales, vulnerabilidad, riesgo

\section{AUTOR}

\section{FERNANDO ANTONIO IGNACIO GONZÁLEZ}

Instituto de Investigaciones Económicas y Sociales del Sur, UNS-CONICET. San Andrés 800, Bahía Blanca-Argentina 